\title{
Monolithically Integrated QCSE-tuned InGaAsP MQW ridge waveguide DBR laser
}

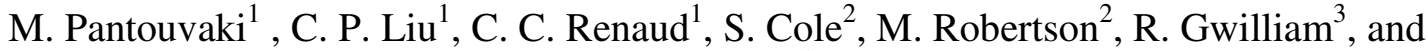 \\ A. J. Seeds ${ }^{1}$ \\ ${ }^{1}$ Department of Electronic and Electrical Engineering, University College London, Torrington \\ Place, London, WC1E 7JE, United Kingdom. \\ ${ }^{2}$ Centre for Integrated Photonics, Adastral Park, Martlesham Heath, Ipswich, Suffolk, IP5 3RE, \\ United Kingdom. \\ ${ }^{3}$ Surrey Ion Beam Centre, Advanced Technology Institute, University of Surrey, Guildford, \\ Surrey, GU2 7XH, United Kingdom.
}

\begin{abstract}
The first QCSE-tuned ridge waveguide InGaAsP MQW DBR laser monolithically integrated with QWintermixed tuning sections is demonstrated. QCSE is used for tuning with minimal thermal effects over $\sim 7 \mathrm{~nm}$ for $<2.5 \mathrm{~V}$ applied voltage change.
\end{abstract}

\section{Introduction}

Tunable lasers are important components for applications such as wavelength division multiplexed transmission and optical switching in optical networks. Wide tuning ranges and high optical power have been demonstrated in devices integrated with distributed Bragg reflectors (DBRs) for wavelength-selectivity $(1,2)$. In these applications, wavelength tuning is typically accomplished by current injection in the tuning sections to induce refractive index changes and select the wavelength from the laser gain spectrum. Alternatively, tuning can be obtained by fieldinduced effects, using the Franz-Keldysh $(3,4)$ or the quantum confined Stark effect (QCSE) $(5,6)$. These techniques have the advantage that the switching speed is not limited by the carrier lifetime, and has therefore the potential for faster switching (4), while the reduced amount of current in the cavity can reduce the thermal effects. In earlier work, we demonstrated QCSE tuning in an external cavity tunable laser (5), and in a monolithically integrated GaAs/AlGaAs laser without DBR (6). In this paper, a monolithically integrated QCSE-tuned InGaAsP MQW ridge waveguide DBR laser is presented for the first time. The laser includes a phase and a DBR section that have been post-growth quantum well intermixed to reduce losses. Noncontinuous tuning over $7 \mathrm{~nm}$ is demonstrated by varying the mirror reverse bias from $0 \mathrm{~V}$ to $-2.5 \mathrm{~V}$, with a side-mode suppression ratio (SMSR) of $\sim 20 \mathrm{~dB}$. Significant improvements in the tuning range and SMSR can be achieved by improving the mirror design (2).

\section{Device description}

Fig. 1 shows the schematic cross-section of the device. The structure was grown by metal-organic vapour phase epitaxy (MOVPE) on a semi-insulating InP substrate. The active region consists of eight $7 \mathrm{~nm}$-wide compressively strained (1\%) InGaAsP Q1.65 wells, alternating with seven $14 \mathrm{~nm}$-wide tensile strained (0.5\%) InGaAsP Q1.3 barriers, sandwiched between two 10nm-thick InGaAsP Q1.3 waveguide layers. Following the active region, a 170nm-InP spacer layer and 30nm Q1.3 etch-stop layers were grown. A first-order grating was then written using electron beam lithography and reactive ion etching, and the structure was completed by an MOVPE overgrowth of $1.4 \mu \mathrm{m}$-thick pdoped InP, a $0.2 \mu \mathrm{m}$ InGaAs contact layer, a $0.5 \mu \mathrm{m} \mathrm{InP}$ buffer layer and a final $0.1 \mu \mathrm{m}$ InGaAs buffer layer.

In order to reduce the losses in the passive sections, quantum-well intermixing using shallow ion implantation was performed. The wafer was masked with $0.6 \mu \mathrm{m} \mathrm{SiO}_{2}$ and the phase and DBR sections were implanted with $8 \times 10^{14} \mathrm{~cm}^{-2}$ $\mathrm{P}^{+}$ions at $100 \mathrm{keV}$ and $200^{\circ} \mathrm{C}$ temperature. After 
implantation, rapid thermal annealing at $650^{\circ} \mathrm{C}$ for $90 \mathrm{~s}$ was used to produce $\sim 35 \mathrm{~nm}$ blueshift of the photoluminescence (PL) of the implanted regions relative to the gain section PL, as shown in Fig. 2.
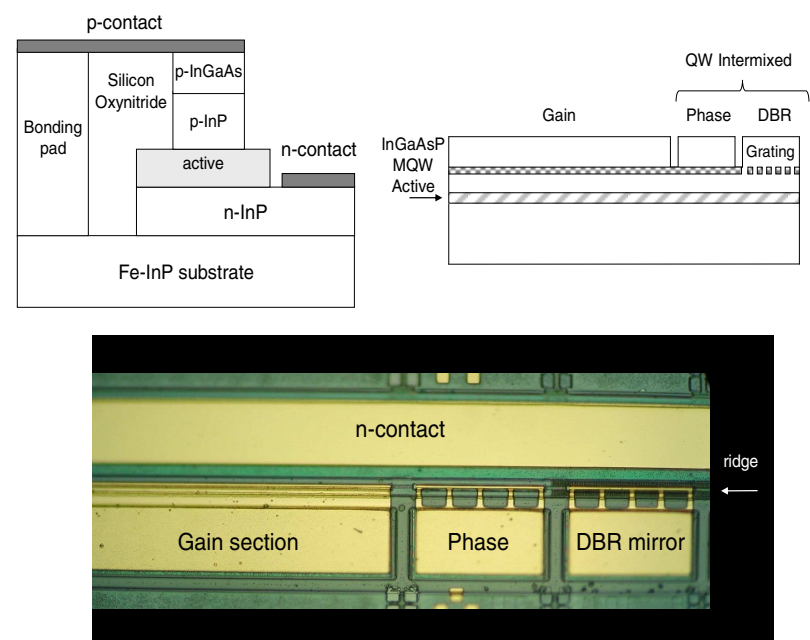

Fig. 1: Schematic cross-section of the QCSE-tuned laser and micrograph of fabricated device.

Following the quantum well intermixing, the top InGaAs and InP buffer layers were removed, and the material was processed into three-section $3.5 \mu \mathrm{m}$-wide ridge waveguide devices, using standard techniques. Electrical isolation between the sections was accomplished by etching the top pInGaAs and p-InP layers, which resulted in $\sim 15 \mathrm{k} \Omega$ resistance between neighbouring p-contacts. Prior to metallization, one side of the ridge was etched to the n-InP layer to form the n-contact, while the other was etched to the semi-insulating InP layer to isolate the bonding pads. This approach reduces the device capacitance. A thick silicon oxynitride layer was deposited and subsequently etched on the ridge and $\mathrm{n}$-InP to provide contact windows. Au-contacts were deposited by sputtering and then etched to form $10 \mu \mathrm{m}$ wide bridges supported by the silicon oxynitride layer, connecting the ridge to the bonding pads. The samples were then thinned to $\sim 100 \mu \mathrm{m}$ and cleaved to form devices with a $1 \mathrm{~mm}$ long active section, a $170 \mu \mathrm{m}$ long phase section and a $200 \mu \mathrm{m}$ DBR mirror, as shown in Fig. 1. The devices were tested without antireflection coatings.

\section{Experimental results}

The tuning characteristics of the device with constant current on the gain section $(I=150 \mathrm{~mA})$, are shown in Fig. 3, where the output wavelength measured from the DBR section facet is plotted versus the DBR section reverse bias. Tuning over $\sim 7 \mathrm{~nm}$ was accomplished by changing the bias on the DBR section from $0 \mathrm{~V}$ to $-2.5 \mathrm{~V}$ and adjusting the phase section reverse bias (from $0 \mathrm{~V}$ to $-0.7 \mathrm{~V}$ ) to maximise the side-mode suppression ratio. As the mirror and phase section reverse bias increased further, the QCSE-induced absorption red-shift resulted in increased absorption at the wavelength of operation, suppressing lasing. The discontinuities in tuning are attributed to parasitic internal reflections at the interfaces of the sections. Fig. 4 shows examples of the optical spectra, where the coupled cavity effects can be observed. The side-mode suppression ratio is $\sim 20 \mathrm{~dB}$, due to weak mirror selectivity. The performance could be improved by using implantation to isolate neighbouring sections and reduce internal reflections, by increasing the DBR mirror coupling factor to improve the SMSR, and by introducing different bandgap detuning in the phase and DBR sections.

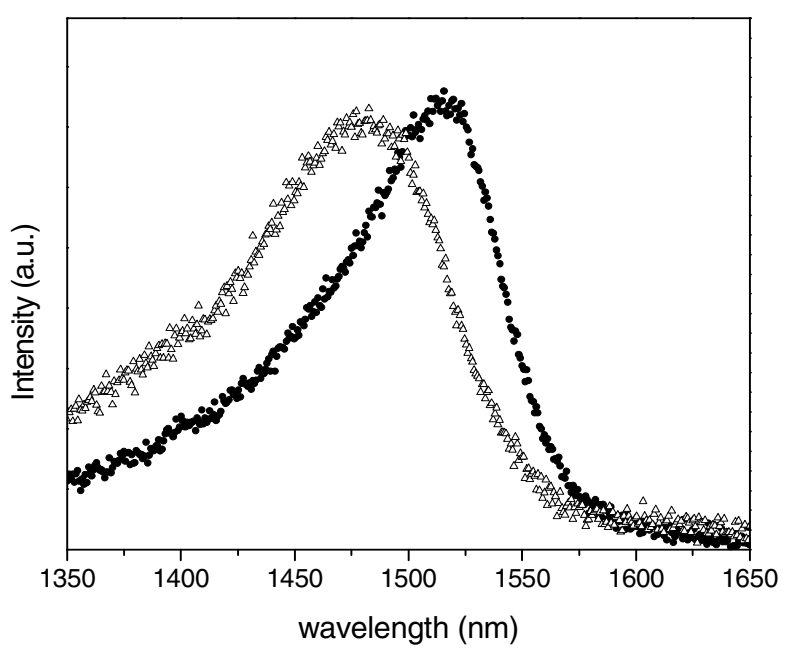

Fig. 2: Photoluminescence spectra of the gain (circles) and tuning (triangles) sections after implantation and annealing at $650^{\circ} \mathrm{C}$ for $90 \mathrm{~s}$.

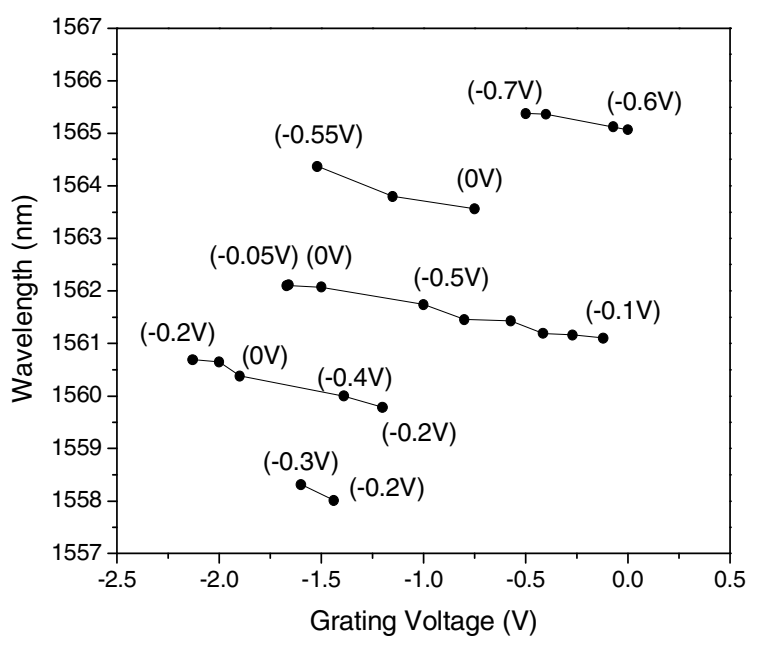

Fig. 3: Laser wavelength versus the reverse bias applied to the mirror section. The phase voltage is shown in brackets. 

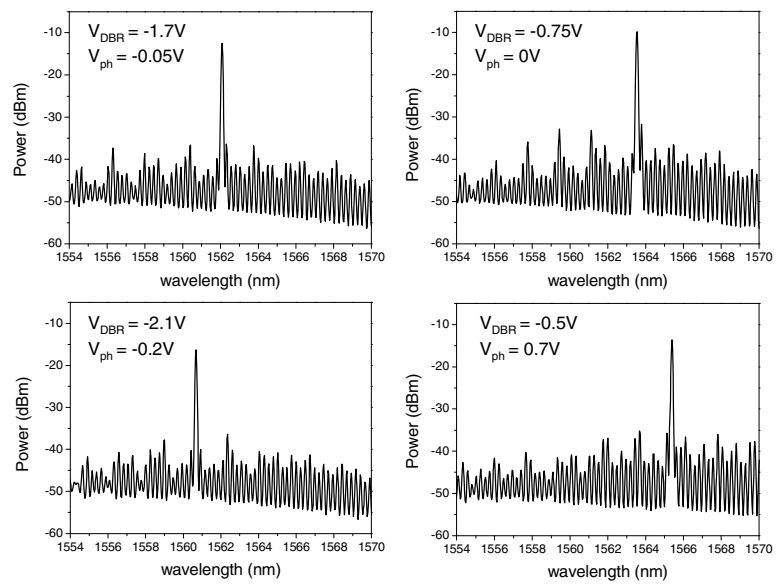

Fig. 4: Laser spectra of the integrated $Q W$ intermixed device, showing the SMSR under different reverse bias conditions on the DBR mirror $\left(V_{D B R}\right)$ and the phase section $\left(V_{p h}\right)$.

\section{Conclusions}

In conclusion, QCSE has been used to demonstrate wavelength tuning in a monolithically integrated InGaAsP MQW ridge waveguide DBR laser for the first time. Postgrowth QW-intermixing based on shallow ion implantation reduced the absorption loss in the tuning and phase sections, while still allowing for QCSE-induced refractive index changes over the operating wavelength range, resulting in non-continuous tuning across $\sim 7 \mathrm{~nm}$ when the mirror reverse bias was varied from $0 \mathrm{~V}$ to $-2.5 \mathrm{~V}$. The device is promising for reducing switching times and thermal effects.

\section{References}

[1] H. Debregeas-Sillard, A. Vuong, F. Delorme, J. David, V. Allard, A. Bodere, O. LeGouezigou, F. Gaborit, J. Rotte, M. Goix, V. Voiriot, and J. Jacquet, "DBR module with 20$\mathrm{mW}$ constant coupled output power, over $16 \mathrm{~nm}(40 \times 50-\mathrm{GHz}$ spaced channels)," IEEE Photon. Technol. Lett., Vol. 13, pp. 4-6, 2001.

[2] V. Jayaraman, Z.-M. Chuang, and L. A. Coldren, "Theory, design and performance of extended tuning range semiconductor lasers with sampled gratings," IEEE J. of Quantum Electron., Vol. 29, pp. 1824-1834, 1993.

[3] T. Wolf, S. Illek, J. Rieger, B. Borchert, and M.-C. Amann, "Tunable twin-guide (TTG) distributed feedback (DFB) laser with over 10nm continuous tuning range," Electron. Lett., Vol. 29, pp. 2124-2125, 1993.

[4] F. Delorme, S. Slempkes, A. Ramdane, B. Rose, and H. Nikajima, "Subnanosecond tunable Distributed Bragg Reflector lasers with an electrooptical Bragg section," IEEE
J. Select. Top. Quantum Electron., Vol. 1, pp. 396-400, 1995.

[5] B. Cai, A. J. Seeds, A. Rivers, J. S. Roberts, "Multiple quantum well tuned GaAs/AlGaAs laser," Electron. Lett., Vol. 25, pp. 145-146, 1989.

[6] X. Huang, A. J. Seeds, and J. S. Roberts, "Reverse bias tuned multiple quantum well ridge guide laser with uniform frequency modulation response," Appl. Phys. Lett., Vol. 71, pp. 765-766, 1997. 